\title{
Minimizing Glycemic Fluctuations in Patients with Type 2 Diabetes: Approaches and Importance
}

\author{
Paresh Dandona, MD, PhD
}

\begin{abstract}
Glycemic fluctuations, characterized by short-term oscillations in plasma glucose, are important when managing type 2 diabetes (T2D) and may be considered a target of glucose-lowering therapies. Continuous glucose monitoring (CGM) has been used to evaluate the effects of different treatments on glycemic fluctuations. This review examines approaches to and the importance of minimizing glycemic fluctuations among patients with T2D. Measures of $\mathrm{HbA}_{1 \mathrm{c}}$, fructosamine, and glycated albumin reflect a long-term average of plasma glucose, and are therefore unable to provide an accurate measure of short-term glycemic oscillations. CGM provides accurate monitoring of real-time glucose fluctuations and has been used to investigate the effects of lifestyle and treatment on daily glycemic control. Dipeptidyl peptidase-4 inhibitors, sodium-glucose cotransporter 2 inhibitors, and glucagon-like peptide-1 receptor agonists have demonstrated significant improvements in measures such as the mean amplitude of glucose excursions and standard deviation of CGM. Case studies of two patients with T2D utilizing CGM are also included in this review, which demonstrated that CGM was a useful tool for diagnosing unrecognized hypoglycemia and hyperglycemia in situations in which it was impractical to check fingerstick concentrations. Altogether, the evidence suggests that glycemic fluctuations are a potential target to consider when managing T2D. CGM allows for the real-time evaluation of glycemic fluctuations and may assist in the development of an individualized treatment plan to adequately control short-term oscillations in blood glucose levels.
\end{abstract}

Keywords: Glucose, Glycemic control, Glycemic fluctuations, Glycemic variability, Type 2 diabetes.

\section{Introduction}

$\mathbf{E}$ FFECTIVELY CONTROLLING BLOOD glucose levels is an important aspect of clinical care in the long-term management of diabetes (both type 1 diabetes [T1D] and type 2 diabetes [T2D]) because it reduces the risk of microvascular complications. ${ }^{1-3}$ However, short-term changes in blood glucose levels, termed glycemic fluctuations, can impact mood and quality of life (QoL). ${ }^{4}$ Glycemic fluctuations may also contribute to the development of vascular complications in patients with $\mathrm{T} 2 \mathrm{D}$, which remain the leading cause of morbidity and mortality in this patient population. ${ }^{5}$ Glycemic fluctuations have traditionally been studied in the context of insulin-treated T1D, but recent research and technology have demonstrated the benefit of monitoring and controlling gly- cemic fluctuations in T2D as well. This review discusses the importance of glycemic control and glycemic fluctuations in the management of patients with T2D.

\section{Glycated Hemoglobin: Can We Do Better Than $\mathrm{HbA}_{1 \mathrm{c}}$ ?}

$\mathrm{HbA}_{1 \mathrm{c}}$ is a measure of the fraction of glycated hemoglobin, which increases with average plasma glucose. As the lifespan of red blood cells is $\sim 4$ months, $\mathrm{HbA}_{1 \mathrm{c}}$ reflects average blood glucose levels over the past 2-3 months. $\mathrm{HbA}_{1 \mathrm{c}}$ is widely used to assess long-term glycemic control in patients with T2D and has been the focus of many glucose-lowering therapies whereby the therapeutic goal is to achieve target $\mathrm{HbA}_{1 \mathrm{c}}$ levels of $<7.0 \%$ or $\leq 6.5 \%$, as recommended by international guidelines. ${ }^{6-8}$ However, $\mathrm{HbA}_{1 \mathrm{c}}$ changes slowly in response to

Department of Medicine, School of Medicine and Biomedical Sciences, State University of New York at Buffalo, Buffalo, New York, USA.

(C) Paresh Dandona, 2017; Published by Mary Ann Liebert, Inc. This Open Access article is distributed under the terms of the Creative Commons Attribution Noncommercial License (http://creativecommons.org/licenses/by-nc/4.0/) which permits any noncommercial use, distribution, and reproduction in any medium, provided the original author(s) and the source are credited. 
treatment, and conditions such as hemolytic or hemorrhagic anemia, kidney disease, liver disease, blood transfusions, and altered red blood cell lifespan can affect its validity. ${ }^{9,10}$

Fructosamine and glycated albumin provide an index of glucose control over 2-3 weeks, rather than the 3-month average provided by $\mathrm{HbA}_{1 \mathrm{c}} \cdot{ }^{11}$ Therefore, these measures may be a preferable alternative to $\mathrm{HbA}_{1 \mathrm{c}}$, particularly in patients with chronic kidney disease who undergo hemodialysis, which has been shown to reduce $\mathrm{HbA}_{1 \mathrm{c}}$ values. ${ }^{12}$ A study by Selvin et al. demonstrated that glycated albumin and fructosamine were strongly associated with microvascular complications among patients with $\mathrm{T} 2 \mathrm{D}$, and associations persisted after adjusting for $\mathrm{HbA}_{1 \mathrm{c}}{ }^{13}$ However, fructosamine and glycated albumin measurements have limitations as well; fructosamine measurements in patients with abnormal albumin turnover and hypoalbuminemia may be inaccurate, ${ }^{11}$ and glycated albumin levels are affected by dysregulated albumin metabolism and hypoalbuminemia. ${ }^{11}$

\section{Glycemic Fluctuations: A Better Picture of Glycemic Control?}

$\mathrm{HbA}_{1 \mathrm{c}}$, fructosamine, and glycated albumin all reflect average blood glucose levels over weeks or months. However, in patients with T2D, blood glucose oscillations can occur within a single day. These short-term oscillations are termed glycemic fluctuations or variability. Specifically, glycemic fluctuations are characterized by variations in blood glucose levels (glycemic excursions) and include hypoglycemic events and postprandial hyperglycemia. ${ }^{14}$ Since $\mathrm{HbA}_{1 \mathrm{c}}$ does not reflect these short-term oscillations, patients with T2D may achieve a target $\mathrm{HbA}_{1 \mathrm{c}}$ while still experiencing marked glycemic fluctuations.

Short-term changes in blood glucose can impact several functions. During acute hyperglycemia (mean blood glucose of $301 \mathrm{mg} / \mathrm{dL}$ ), 20 adults with T2D were found to have impaired performance on tests of information processing, working memory, and attention. ${ }^{15}$ Tension increased during acute hyperglycemia, while hedonic tone and energetic arousal (feelings of happiness and alertness, respectively) decreased. Furthermore, for 45 patients with T2D, the rate of change of postmeal blood glucose was shown to correlate with depressive and anxious mood and symptoms of cognitive dysfunction. ${ }^{16}$

Glycemic fluctuations are also associated with QoL and patient-reported outcomes. In patients with T1D $(n=82)$ and insulin-treated T2D $(n=306)$, reductions in intraday mean glucose, glycemic fluctuations, and excursions above $140 \mathrm{mg} / \mathrm{dL}$, as measured by continuous glucose monitoring (CGM), were associated with improvements in patient satisfaction and perceived health, independent of concurrent reductions in daily glucose levels and $\mathrm{HbA}_{1 \mathrm{c}} \cdot{ }^{17}$ In patients with $\mathrm{T} 2 \mathrm{D}(n=54)$ initiating insulin pump therapy, a reduction in glycemic fluctuations was associated with increased treatment preference and improved health utility (a measure of QoL based on mobility, self-care, activities, pain/discomfort, and anxiety/depression), which the authors believe may lead to enhanced treatment adherence. ${ }^{18}$ Across treatments, some physicians and patients believe that better QoL may potentially improve adherence to treatment, ${ }^{19}$ whereas others feel that enhanced treatment adherence may contribute to an improved QoL. ${ }^{20,21}$ Therefore, control of short-term changes in blood glucose has important effects on a patient's day-to-day life and may contribute to a positive feedback loop of improved glycemic control, treatment adherence, and QoL.

Glycemic fluctuations also play a role in the long-term management of diabetic complications. In a recent editorial, Hirsch $^{22}$ noted that, as the treatment of T1D (and often patients with a long duration of T2D) shifted from once- or twice-daily insulin to multiple daily injections or a continuous subcutaneous insulin infusion, approaches that more closely mimicked physiologic insulin concentrations and likely reduced glycemic oscillations, the occurrence of microvascular complications declined. Hirsch hypothesized this was an early indicator that glycemic fluctuations may be related to diabetic complications.

This is further supported in a review by $\mathrm{Yu}$ et al., which suggests that postprandial hyperglycemia-a key contributor to glycemic fluctuations - is a risk factor for macrovascular complications, and its control is important in reducing the risk of developing cardiovascular disease in patients with T2D. ${ }^{5}$ Specifically, in a study of over 20,000 patients from 10 prospective European cohort trials, the DECODE study group found an increased risk of death from cardiovascular disease and all causes in patients with high 2-h glucose concentrations (impaired glucose tolerance [140-198 mg/dL] and diabetes $[\geq 198 \mathrm{mg} / \mathrm{dL}])$ following an oral glucose tolerance test. ${ }^{23}$ Another report of 3370 patients from the Framingham Offspring Study found that the 2-h response to an oral glucose challenge was associated with cardiovascular events, independent of other cardiovascular risks and measures of hyperglycemia. ${ }^{24}$ In addition, a meta-analysis of 13 studies in patients with T2D found that long-term glycemic fluctuations, measured by the coefficient of variation or standard deviation (SD) of $\mathrm{HbA}_{1 \mathrm{c}}$, were associated with renal and cardiovascular disease, macrovascular events, and mortality, but not with microvascular events. ${ }^{25}$

However, contrary to the above-cited studies, some suggest that there is no evidence for a relationship between glycemic fluctuations and diabetic complications. A reanalysis of the Diabetes Control and Complications Trial, which initially suggested the risk for microvascular complications may be related to postprandial hyperglycemia, found these results to be an artifact of the statistical models used. ${ }^{26}$ Instead, the risk for complications was primarily determined by $\mathrm{HbA}_{1 \mathrm{c}}$. Although more research is necessary, glycemic fluctuations have been recommended as an emerging target in the management of diabetes $^{27}$ and may potentially impact the risk of complications in patients who have otherwise achieved $\mathrm{HbA}_{1 \mathrm{c}}$ targets.

\section{Glycemic Fluctuations and Oxidative Stress}

Acute hyperglycemic fluctuations can potentially contribute to diabetic complications through increased glycation and activation of oxidative stress. ${ }^{28}$ The induction of reactive oxygen species (ROS) generation and lipid peroxidation by glucose and saturated fat was first demonstrated by Mohanty et al. ${ }^{29,30}$ This work was developed further by the demonstration that a high-fat, high-calorie mixed meal induced oxidative and inflammatory stress for several hours. ${ }^{31}$ Thus, even in healthy subjects, the intake of macronutrients can induce periodic oxidative and inflammatory stress, as reflected in increased ROS generation, lipid peroxidation, and the induction of endotoxemia and proinflammatory cytokines. $^{32}$ These effects can potentially be amplified in patients 
with diabetes and chronic hyperglycemia, who have increased basal levels of oxidative and inflammatory stress. ${ }^{33}$

Monnier et al. investigated the relationship between chronic hyperglycemia, acute glucose fluctuations, and oxidative stress in patients with T2D and found a significant relationship between acute glucose fluctuations and oxidative stress. ${ }^{34}$ This case-controlled study used CGM to measure fasting plasma glucose and $\mathrm{HbA}_{1 \mathrm{c}}$; mean amplitude of glucose excursions (MAGE) and postprandial area under the curve (AUC) were used to measure acute glucose fluctuations and postprandial hyperglycemia, respectively. Oxidative stress was measured using 24-h urinary excretion of free 8-iso-prostaglandin F2 $\alpha$. Oxidative stress is mediated by increased ROS production at the mitochondrial level and membrane NADPH oxidase activity. These mechanisms link persistent hyperglycemia and acute glucose fluctuations with glucose-induced activation of protein kinase $\mathrm{C}$ isoforms, an increase in p38 mitogenactivated protein kinase, ${ }^{35}$ increased production of advanced glycation end products, and greater glucose flow through the aldose reductase pathway-all of which lead to damage of vascular endothelial cells in diabetes. ${ }^{36}$ Acute glucose load and hyperglycemia also induce ROS generation through NADPH oxidase-mediated mechanisms. ${ }^{29,37}$

\section{Glucose Monitoring Increases Awareness of Glycemic Fluctuations}

Glucose monitoring can increase patients' awareness of the relationship between their lifestyle and daily glycemic fluctuations. For instance, in a study of 201 insulin-treated patients with $\mathrm{T} 2 \mathrm{D}, \mathrm{HbA}_{1 \mathrm{c}}$ was significantly decreased by $0.30 \%$ and $0.36 \%$ after 4 and 8 weeks, respectively, of selfmonitored blood glucose (SMBG). ${ }^{38}$ Compliance was a strong predictor of the improvement at 8 weeks, after adjusting for baseline $\mathrm{HbA}_{1 \mathrm{c}}$. However, compliance ranged from $3 \%$ to $100 \%$, with an average of $78.4 \%$.

CGM offers a potential alternative to SMBG for patients amenable to the CGM system. Recent advances in CGM technology have made the use of CGM systems easier than past versions, and integration of small CGM systems into closed-loop insulin delivery systems is underway. Future aims for CGM technology include further miniaturization of CGM systems. This easy-access, real-time monitoring system of glucose levels, which also alerts patients to high or low glucose values, can reduce glycemic fluctuations by improving patients' awareness of hyperglycemia and hypoglycemia. ${ }^{39}$

\section{CGM to Evaluate Effectiveness of Treatments on Glycemic Fluctuations}

CGM provides a tool for evaluating the impact of different treatments on glycemic control. In a 4-week study in 33 patients with T2D who had inadequate glycemic control after $\geq 2$ months on metformin, similar $\mathrm{HbA}_{1 \mathrm{c}}$ reductions were seen among patients assigned to treatment with the dipeptidyl peptidase-4 (DPP-4) inhibitor sitagliptin (from a mean of $7.0 \%$ to $6.6 \% ; P<0.001)$ and those assigned to the sulfonylurea glimepiride (from a mean of $7.3 \%$ to $6.9 \% ; P<0.001$ ). ${ }^{40}$ However, a significant reduction in MAGE was only seen in the sitagliptin group (from 88 to $67 \mathrm{mg} / \mathrm{dL} ; P<0.001$ ). The greater impact of sitagliptin on reducing glucose fluctuations may be related to the glucose-dependent mechanism of action for glucose-lowering effects, whereas sulfonylureas, which are not sensitive to blood glucose concentrations, increase the risk of blood glucose troughs that may contribute to fluctuations (Table 1).

Sitagliptin and vildagliptin add-on therapy for 8 weeks resulted in similar, significant $(P<0.05)$ improvements from baseline in 24-h glucose fluctuations (as indicated by reductions of $\sim 15 \mathrm{mg} / \mathrm{dL}$ in MAGE) in patients with T2D inadequately controlled by metformin. ${ }^{41}$ However, only vildagliptin recipients had significant $(P<0.05)$ improvements from baseline in parameters reflecting daily glycemic control (mean 24-h blood glucose level, time spent in the ideal glycemic range [70$140 \mathrm{mg} / \mathrm{dL}]$ and above hyperglycemic thresholds [140 and $180 \mathrm{mg} / \mathrm{dL}$ ], along with corresponding AUC values).

The $\alpha$-glucosidase inhibitors are a class of glucoselowering drugs taken at meals that specifically reduce postprandial glucose by inhibiting carbohydrate digestion in the small intestine and delaying its absorption (Table 1). ${ }^{42} \mathrm{~A}$ 24-week randomized controlled trial compared the addition of the $\alpha$-glucosidase inhibitor acarbose or the sulfonylurea glibenclamide to metformin in patients with poorly controlled T2D $(n=55) .{ }^{43}$ While both treatments significantly reduced $\mathrm{HbA}_{1 \mathrm{c}}(P<0.001)$, the addition of acarbose significantly decreased MAGE (from a mean of 101 to $72 \mathrm{mg} / \mathrm{dL}$; $P<0.001$ ), whereas glibenclamide had no significant effect on MAGE (mean of $112 \mathrm{mg} / \mathrm{dL}$ at baseline and $114 \mathrm{mg} / \mathrm{dL}$ at study end). Likewise, among 45 patients with T2D who had high CGM MAGE values $(\geq 61 \mathrm{mg} / \mathrm{dL})$ on premixed insulin analogue therapy, the addition of acarbose significantly decreased MAGE (from 77 to $47 \mathrm{mg} / \mathrm{dL} ; P<0.01$ ) and the mean of daily differences (from 23 to $20 \mathrm{mg} / \mathrm{dL} ; P<0.05$ ), a measure of interday glycemic fluctuations. ${ }^{44}$ The incidence of hypoglycemic events in these patients decreased from $24 \%$ to $2 \%(P<0.01)$.

In addition, CGM was utilized in a 4-week, randomized, placebo-controlled study to evaluate the sodium-glucose cotransporter 2 inhibitor dapagliflozin in 100 adult patients with T2D also being treated with either metformin alone or insulin and up to two oral glucose-lowering drugs. ${ }^{45}$ At day 28 , dapagliflozin $10 \mathrm{mg} /$ day significantly reduced MAGE compared with placebo $(-10.0$ vs. $+5.3 \mathrm{mg} / \mathrm{dL} ; P=0.01)$. Furthermore, dapagliflozin significantly reduced the proportion of time with blood glucose $>180 \mathrm{mg} / \mathrm{dL}(-12.6 \%$ vs. $+3.5 \% ; P<0.001)$ and increased the proportion of time spent with blood glucose levels in the target range of $70-180 \mathrm{mg} / \mathrm{dL}(+12.2 \%$ vs. $-2.8 \% ; P<0.001)$. A small increase in the proportion of time with blood glucose $<70 \mathrm{mg} / \mathrm{dL}(+0.3 \%$ vs. $-0.6 \%)$ was driven by the dapagliflozin plus insulin group.

CGM can also uncover hypoglycemia, even when no symptoms are present. ${ }^{46}$ In a study of 29 patients with T2D well controlled on metformin \pm sulfonylureas, 18 patients (62\%) experienced 65 silent, symptom-free hypoglycemic episodes (interstitial glucose $\leq 70 \mathrm{mg} / \mathrm{dL}$ ). CGM can alert patients to these silent changes in blood glucose levels, providing patients with the opportunity to make the necessary adjustments to potentially avoid these episodes.

For those patients who require additional therapy beyond oral agents, insulin and other injectables such as glucagon-like peptide-1 receptor agonists (GLP-1RAs) are used. Small pilot and subpopulation studies first provided evidence for glucose fluctuation-lowering effects of GLP-1RAs, ${ }^{47,48}$ whereas larger studies designed to assess the effects of GLP-1RAs on glucose fluctuations have been carried out more recently. 


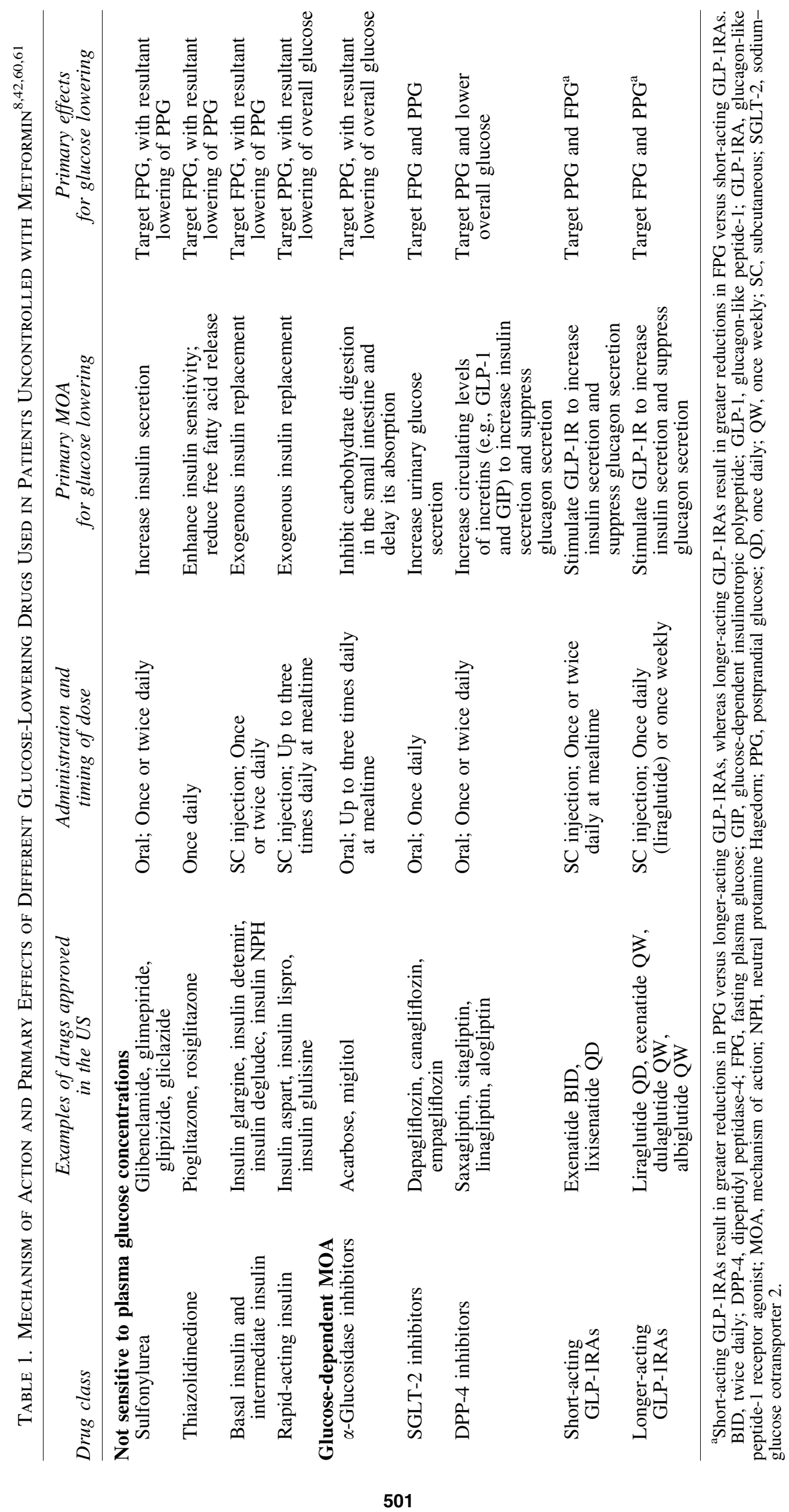


FLAT-SUGAR was a 26-week study comparing the effects on glycemic fluctuations (measured using CGM) of the GLP-1RA exenatide twice daily (BID) or rapid-acting insulin as prandial add-on therapy to insulin glargine plus metformin in patients with T2D $(n=102){ }^{49}$ While exenatide BID and prandial insulin both target postprandial glucose concentrations, the insulinogenic effects of exenatide BID are glucose dependent, in contrast to insulin (Table 1). After 26 weeks of treatment, $\mathrm{HbA}_{1 \mathrm{c}}$ levels were comparable in the exenatide BID and rapidacting insulin groups $(7.1 \%$ and $7.2 \%$, respectively). Mean changes in the coefficients of variation of glucose from baseline to 26 weeks (as determined by CGM) were significantly different between the exenatide BID and rapid-acting insulin groups $(-2.4$ vs. $+0.4 ; P=0.047)$. Furthermore, MAGE was improved in the exenatide BID group compared with the rapidacting insulin group $(P=0.049)$.

In a recent, randomized, double-blind study, patients with T2D treated with exenatide once weekly (QW) and metfor$\min (n=60)$ had improvements in daily glycemic control and reductions in glucose fluctuations compared with patients treated with placebo and metformin $(n=56) .{ }^{50}$ Exenatide QW was associated with reductions from baseline in 24-h glucose profiles at weeks 4 and 10, whereas changes with placebo were less pronounced (Fig. 1). Compared with placebo, exenatide QW significantly reduced 24-h mean glucose $(-30.8$ vs. $-3.0 \mathrm{mg} / \mathrm{dL})$, MAGE $(-15.2 \mathrm{vs} .+2.9 \mathrm{mg} / \mathrm{dL})$, and the SD of mean glucose ( $-6.3 \mathrm{vs} .+0.7 \mathrm{mg} / \mathrm{dL}$ ) at week 10 (all $P<0.001)$. In addition, exenatide $\mathrm{QW}$ significantly reduced the proportion of time spent with patient blood glucose concentrations in the hyperglycemic range $(>180 \mathrm{mg} / \mathrm{dL}$; $P<0.001)$ and significantly increased the proportion of time spent in the target glycemic range $(70-180 \mathrm{mg} / \mathrm{dL} ; P<0.001)$ compared with placebo, without increasing time spent in the hypoglycemic range $(<70 \mathrm{mg} / \mathrm{dL})$.
In an analysis of three randomized controlled trials comparing insulin glargine (two studies) or insulin detemir (one study) with exenatide QW, treatment with exenatide QW had a greater reduction in $\mathrm{HbA}_{1 \mathrm{c}}$ than either insulin $(-1.5 \%$ vs. $-1.3 \%$ $[P<0.02]$ and $-1.1 \%$ vs. $-0.7 \%[P<0.001]$ for the two studies of insulin glargine; $-1.3 \%$ vs. $-0.9 \%[P<0.001]$ for the study of insulin detemir). ${ }^{51}$ Furthermore, treatment with exenatide QW resulted in a significantly greater reduction in MAGE, as compared with insulin glargine ( -12 vs. $-4 \mathrm{mg} / \mathrm{dL}[P=0.002]$; -27 vs. $-1 \mathrm{mg} / \mathrm{dL}[P<0.001])$, but not compared with insulin detemir $(-16$ vs. $-8 \mathrm{mg} / \mathrm{dL}[P=0.080])$. Similar results were found for blood glucose range and blood glucose variability.

The GLP-1RA liraglutide has also been found to reduce glycemic fluctuations in patients with T2D. For instance, IDegLira - a fixed-ratio combination of insulin degludec and liraglutide - has been shown to reduce prandial glucose. ${ }^{52}$ After 26 weeks of treatment $(n=260)$, a meal test found a significantly $(P=0.0023)$ greater reduction in mean \pm SD postprandial glucose increment with IDegLira $(-15.7 \pm 29.7 \mathrm{mg} / \mathrm{dL})$ as compared with insulin degludec $(-3.1 \pm 35.7 \mathrm{mg} / \mathrm{dL})$; treatment with liraglutide had a comparable decrease $(-14.1 \pm 29.2 \mathrm{mg} / \mathrm{dL})$ to that of IDegLira. When the change from baseline in postprandial interstitial glucose increment was assessed with CGM, IDegLira had a significantly greater effect than insulin degludec (estimated treatment difference: $-6.1 \mathrm{mg} / \mathrm{dL} ; P=0.0047$ ) and a comparable effect to liraglutide (estimated treatment difference: $-1.8 \mathrm{mg} / \mathrm{dL} ; P=0.4122$ ).

A separate study of 63 patients with T2D compared treatment with liraglutide and neutral protamine Hagedorn (NPH) insulin. After 12 weeks, treatment with liraglutide was associated with a significantly $(P<0.05)$ greater decrease in MAGE $(-77.4 \mathrm{mg} / \mathrm{dL}$ with liraglutide; $-46.8 \mathrm{mg} / \mathrm{dL}$ with $\mathrm{NPH}$ insulin) and in large amplitude of glycemic excursions $(-104.4 \mathrm{mg} / \mathrm{dL}$ with liraglutide; $-54.0 \mathrm{mg} / \mathrm{dL}$ with NPH insulin), as assessed by CGM. ${ }^{53} \mathrm{In}$

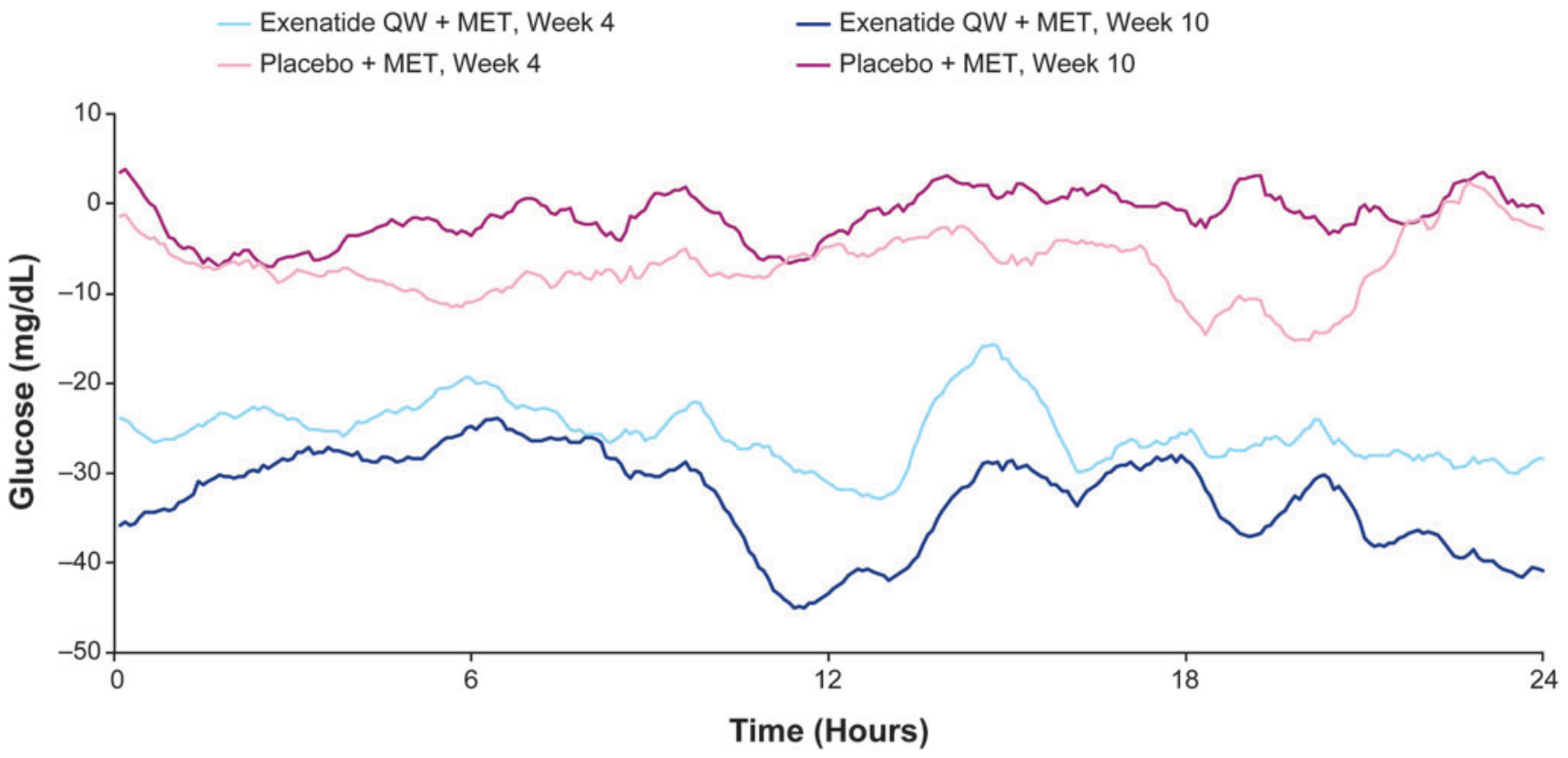

FIG. 1. Change from baseline in mean 24-h glucose profiles at weeks 4 and 10 (exenatide QW + MET, $n=60$; placebo + MET, $n=56$ ). Light blue line = exenatide QW + MET at week 4; navy blue line=exenatide QW + MET at week 10; light pink line $=$ placebo + MET at week 4; magenta line $=$ placebo + MET at week 10. MET, metformin; QW, once weekly. Reproduced with permission from Frías et al. ${ }^{50}$ 
37 patients with T2D treated with either liraglutide plus intensive high-dose insulin therapy or standard insulin uptitration for 6 months, CGM again showed reduced glycemic fluctuations, as measured by the within-day SD of mean daily glucose $(22.5 \%$ reduction with liraglutide plus insulin $[P<0.0001]$; no significant change with standard insulin therapy alone). ${ }^{54}$ An uncontrolled study of 20 Japanese patients with T2D also found liraglutide to significantly reduce MAGE (from 94 to $44 \mathrm{mg} / \mathrm{dL} ; P<0.001$ vs. baseline), as well as the proportion of time spent in hyperglycemia, without any increased time spent in hypoglycemia. 55

Although exenatide BID and liraglutide are both GLP1RAs, they can have differing effects on glycemic fluctuations. An open-label, randomized study of 35 patients with T2D inadequately controlled on sulfonylureas compared the effects of 7 days of treatment with either exenatide BID or liraglutide (once daily) on glycemic fluctuations, as measured by $24-\mathrm{h} \mathrm{CGM}^{56}$ No differences were found for 24 -h mean glucose, SD of blood glucose, MAGE, or early fasting glucose. However, for breakfast and dinner, 3-h postmeal glucose was significantly lower with exenatide BID than with liraglutide $(P<0.01)$, whereas both the mean and SD of blood glucose between 12:00 am and 6:00 am was significantly higher $(P<0.05)$ with exenatide BID compared with liraglutide. This difference may reflect the duration of action and the pharmacodynamics of the two drugs (Table 1).

Finally, as part of the AWARD-4 trial, the effects of dulaglutide versus insulin glargine on glycemic fluctuations were assessed. ${ }^{57}$ A subgroup of 144 patients taking either once-weekly dulaglutide ( 1.5 or $0.75 \mathrm{mg}$ ) or insulin glargine, both added to prandial insulin lispro, were evaluated using CGM. After 26 weeks, dulaglutide $1.5 \mathrm{mg}$ significantly reduced glycemic fluctuations compared with insulin glargine, as measured by within-patient SD (-12.6 vs. $-7.2 \mathrm{mg} / \mathrm{dL}$; $P<0.05)$ and mean of daily differences $(-7.2 \mathrm{vs} .+1.8 \mathrm{mg} / \mathrm{dL}$; $P<0.05)$. At 52 weeks, within-patient SD and mean of daily differences were similar across all groups. At both time points, MAGE was similar across groups.

Data from the above studies demonstrate that multiple glucose-lowering agents that act in a glucose-dependent manner, such as DPP-4 inhibitors and GLP-1RAs, or that inhibit carbohydrate digestion, such as $\alpha$-glucosidase inhibitors, may reduce the occurrence of hyperglycemia without increasing the risk of hypoglycemia. Together, this limits short-term glycemic fluctuations. In addition, DPP-4 inhibitors $^{58}$ and GLP-1RAs ${ }^{59}$ have ROS-suppressive and antiinflammatory effects. As glycemic fluctuations have been linked to oxidative stress and downstream vascular dysfunction and damage, ${ }^{34}$ this may provide further benefit for patients in addition to reducing glycemic fluctuations. Insulin treatments were generally not as effective against glycemic fluctuations, although they were effective in reducing the longer-term measure of glycemic control, $\mathrm{HbA}_{1 \mathrm{c}}$. Additional studies that utilize CGM would be helpful to further establish the effects of these different agents on glycemic fluctuations.

\section{CGM in Clinical Practice: Case Studies}

The two cases below demonstrate that CGM is a useful tool for diagnosing unrecognized hypoglycemia and hyperglycemia in situations in which it is impractical to check fingerstick concentrations.

\section{Case 1}

A 41-year-old male with T2D regularly exercised by running 3-4 miles daily. The patient had good glycemic control with an $\mathrm{HbA}_{1 \mathrm{c}}$ of $6.8 \%$ on treatment with metformin and continuous subcutaneous insulin infusion (i.e., an insulin pump). After his daily run, he reported feeling weakness and a lack of energy. He denied having neurogenic symptoms of hypoglycemia, such as sweating, shakiness, palpitations, anxiety, or increased sensation of hunger. He also denied neuroglycopenic symptoms, such as dizziness, inappropriate behavior, difficulty with concentration, confusion, or blurred vision.

To investigate the possibility of unrecognized hypoglycemia, the patient was fitted with a CGM system and asked to check fingerstick blood glucose at the time of his symptoms. CGM revealed hypoglycemia during the patient's run as well as marked postmeal hyperglycemia. Despite a reduction in basal insulin, he continued to experience hypoglycemia during and after running. However, during weight training (i.e., resistance training exercises), CGM indicated that he maintained normoglycemia. As a result, the patient switched from running to resistance training for his routine exercise. To prevent postmeal hyperglycemia, he increased his dose of prandial bolus insulin, thereby increasing his insulin to carbohydrate ratio factor. Following these changes, the patient's $\mathrm{HbA}_{1 \mathrm{c}}$ fell to $6.5 \%$ without any hypoglycemia or postmeal hyperglycemia. Fluctuations in his blood glucose concentrations were also reduced, leaving the patient feeling more safe and secure while exercising and administering additional bolus insulin doses with meals. Thus, CGM significantly changed the QoL of this patient while enabling him to achieve his $\mathrm{HbA}_{1 \mathrm{c}}$ goal safely, without any hypoglycemia-related complications.

\section{Case 2}

A 52-year-old female with T2D for 8 years reported waking up on several occasions during the night following nightmares that left her sweaty, nervous, and shaky. The patient reported normal fasting blood glucose concentrations. She was on treatment with metformin (1000 mg BID), insulin glargine (30 $\mathrm{U}$ at bed time), and insulin lispro (three times daily before meals). This regimen was initiated in an attempt to intensify her treatment for uncontrolled T2D with an $\mathrm{HbA}_{1 \mathrm{c}}$ of $10.2 \%$. She was $\sim 1$ year postmenopausal and occasionally experienced hot flashes during the day. With estradiol therapy, her daytime hot flashes had resolved, but she continued to have nocturnal sweaty episodes. She was referred to our diabetes and endocrinology clinic for further evaluation of her episodes of night sweats and nervousness, often coupled with nightmares. Her $\mathrm{HbA}_{1 \mathrm{c}}$ was $7.8 \%$ and a random blood glucose concentration was $180 \mathrm{mg} / \mathrm{dL}$. She had mild neuropathy and microalbuminuria.

In an attempt to elucidate the relationship between the nighttime episodes and glycemic levels, the patient was fitted with a CGM system. CGM revealed a consistent fall in blood glucose concentrations after midnight, lasting from $20 \mathrm{~min}$ to $1 \mathrm{~h}$, with the trend beginning at 9:00 pm. One of these hypoglycemic episodes coincided with a nightmare-associated episode of night sweats. A diagnosis of nocturnal hypoglycemia was made, and her doses of insulin lispro with dinner and insulin glargine at bedtime were reduced. The episodes of night sweats and nightmares disappeared. Later, the patient substituted a GLP-1RA for insulin lispro treatment and her 
$\mathrm{HbA}_{1 \mathrm{c}}$ fell to $7.0 \%$ without clinical or CGM-recorded hypoglycemia.

Through CGM, the patients described above were able to check for hypoglycemia and hyperglycemia in situations, such as running or sleeping, that would otherwise be impractical for fingerstick checks. The insight provided by CGM led to an improved understanding of daily blood glucose fluctuations, allowing for targeted lifestyle and therapeutic changes and an improvement in their QoL.

\section{Conclusions}

Glycemic fluctuations, through either chronic sustained hyperglycemia and/or acute glucose variations, may be associated with diabetic complications. $\mathrm{HbA}_{1 \mathrm{c}}$ remains the gold standard for assessing glycemic control and the efficacy of glucose-lowering therapies. However, $\mathrm{HbA}_{1 \mathrm{c}}$ has limitations, such as the inability to reflect the peaks and troughs of blood glucose levels that occur daily in many patients with T2D. Glycemic fluctuations are emerging as an important potential parameter to consider when managing blood glucose levels, with potential effects on the risk of diabetic complications such as cardiovascular disease and QoL.

For certain patients, CGM may be a useful tool for assessing the impact of treatment and lifestyle on daily changes in blood glucose levels. Recent studies have utilized CGM to demonstrate reductions in glycemic fluctuations with glucoselowering therapies, such as sodium-glucose cotransporter 2 inhibitors, DPP-4 inhibitors, and GLP-1RAs, and future CGM studies would provide further insight into the effects of specific treatments on glycemic fluctuations.

\section{Acknowledgments}

Raelene Simpson, on behalf of inScience Communications, Springer Healthcare, and Mollie Marko, PhD, of inScience Communications, Springer Healthcare (New York, NY, USA), provided medical writing support, which was funded by AstraZeneca.

\section{Author Disclosure Statement}

P.D. has received research support and/or funding from AbbVie, Boehringer Ingelheim, Bristol-Myers Squibb, the Endocrine Fellowship Foundation, GlaxoSmithKline, the Juvenile Diabetes Foundation, Merck, the National Institutes of Health, and Novo Nordisk; and honoraria from AbbVie, AstraZeneca, Boehringer Ingelheim, Bristol-Myers Squibb, Eli Lilly and Company, GlaxoSmithKline, Merck, Novo Nordisk, and Sanofi.

\section{References}

1. UK Prospective Diabetes Study (UKPDS) Group: Intensive blood-glucose control with sulphonylureas or insulin compared with conventional treatment and risk of complications in patients with type 2 diabetes (UKPDS 33). Lancet 1998;352:837-853.

2. The Diabetes Control and Complications Trial Research Group: The effect of intensive treatment of diabetes on the development and progression of long-term complications in insulin-dependent diabetes mellitus. The Diabetes Control and Complications Trial Research Group. N Engl J Med 1993;329:977-986.
3. Ohkubo Y, Kishikawa H, Araki E, et al.: Intensive insulin therapy prevents the progression of diabetic microvascular complications in Japanese patients with non-insulindependent diabetes mellitus: a randomized prospective 6-year study. Diabetes Res Clin Pract 1995;28:103-117.

4. Penckofer S, Quinn L, Byrn M, et al.: Does glycemic variability impact mood and quality of life? Diabetes Technol Ther 2012;14:303-310.

5. Yu PC, Bosnyak Z, Ceriello A: The importance of glycated haemoglobin $(\mathrm{HbA}(1 \mathrm{c}))$ and postprandial glucose (PPG) control on cardiovascular outcomes in patients with type 2 diabetes. Diabetes Res Clin Pract 2010;89:1-9.

6. Handelsman Y, Bloomgarden ZT, Grunberger G, et al.: American Association of Clinical Endocrinologists and American College of Endocrinology-clinical practice guidelines for developing a diabetes mellitus comprehensive care plan-2015. Endocr Pract 2015;21(Suppl 1):1-87.

7. American Diabetes Association: Standard of medical care in diabetes-2016. (5) Glycemic targets. Diabetes Care 2016;39(Suppl 1):S39-S46.

8. Inzucchi SE, Bergenstal RM, Buse JB, et al.: Management of hyperglycemia in type 2 diabetes, 2015: a patient-centered approach: update to a position statement of the American Diabetes Association and the European Association for the Study of Diabetes. Diabetes Care 2015;38:140-149.

9. Dandona P, Freedman D, Moorhead JF: Glycosylated haemoglobin in chronic renal failure. Br Med J 1979;1:11831184.

10. Selvin E, Rawlings AM, Lutsey PL, et al.: Fructosamine and glycated albumin and the risk of cardiovascular outcomes and death. Circulation 2015;132:269-277.

11. Wright LA-C, Hirsch IB: The challenge of the use of glycemic biomarkers in diabetes: reflecting on hemoglobin A1C, 1,5-anhydroglucitol, and the glycated proteins fructosamine and glycated albumin. Diabetes Spectr 2012;25: $141-148$.

12. Inaba M, Okuno S, Kumeda $\mathrm{Y}$, et al.: Glycated albumin is a better glycemic indicator than glycated hemoglobin values in hemodialysis patients with diabetes: effect of anemia and erythropoietin injection. J Am Soc Nephrol 2007;18:896903.

13. Selvin E, Rawlings AM, Grams M, et al.: Fructosamine and glycated albumin for risk stratification and prediction of incident diabetes and microvascular complications: a prospective cohort analysis of the Atherosclerosis Risk in Communities (ARIC) study. Lancet Diabetes Endocrinol 2014;2:279-288.

14. Satya Krishna SV, Kota SK, Modi KD: Glycemic variability: clinical implications. Indian $\mathbf{J}$ Endocrinol Metab 2013;17:611-619.

15. Sommerfield AJ, Deary IJ, Frier BM: Acute hyperglycemia alters mood state and impairs cognitive performance in people with type 2 diabetes. Diabetes Care 2004;27:23352340.

16. Cox DJ, McCall A, Kovatchev B, et al.: Effects of blood glucose rate of changes on perceived mood and cognitive symptoms in insulin-treated type 2 diabetes. Diabetes Care 2007;30:2001-2002.

17. Testa MA, Gill J, Su M, et al.: Comparative effectiveness of basal-bolus versus premix analog insulin on glycemic variability and patient-centered outcomes during insulin intensification in type 1 and type 2 diabetes: a randomized, controlled, crossover trial. J Clin Endocrinol Metab 2012; 97:3504-3514. 
18. Peyrot M, Rubin RR, Chen X, et al.: Associations between improved glucose control and patient-reported outcomes after initiation of insulin pump therapy in patients with type 2 diabetes mellitus. Diabetes Technol Ther 2011;13:471476.

19. Evans M, Jensen HH, Bogelund M, et al.: Flexible insulin dosing improves health-related quality-of-life (HRQoL): a time trade-off survey. J Med Econ 2013;16:1357-1365.

20. Chew BH: Medication adherence on quality of life among adults with type 2 diabetes mellitus: an exploratory analysis on the EDDMQoL study. Qual Life Res 2015;24:27232731.

21. Polonsky WH, Fisher L, Hessler D, et al.: Patient perspectives on once-weekly medications for diabetes. Diabetes Obes Metab 2011;13:144-149.

22. Hirsch IB: Glycemic variability and diabetes complications: does it matter? Of course it does! Diabetes Care 2015;38:1610-1614.

23. DECODE Study Group, the European Diabetes Epidemiology Group: Glucose tolerance and cardiovascular mortality: comparison of fasting and 2-hour diagnostic criteria. Arch Intern Med 2001;161:397-405.

24. Meigs JB, Nathan DM, D’ Agostino RB, Sr., et al.: Fasting and postchallenge glycemia and cardiovascular disease risk: the Framingham Offspring Study. Diabetes Care 2002;25:1845-1850.

25. Gorst C, Kwok CS, Aslam S, et al.: Long-term glycemic variability and risk of adverse outcomes: a systematic review and meta-analysis. Diabetes Care 2015;38:2354-2369.

26. Lachin JM, Genuth S, Nathan DM, et al.: Effect of glycemic exposure on the risk of microvascular complications in the diabetes control and complications trial-revisited. Diabetes 2008;57:995-1001.

27. Frontoni S, Di Bartolo P, Avogaro A, et al.: Glucose variability: an emerging target for the treatment of diabetes mellitus. Diabetes Res Clin Pract 2013;102:86-95.

28. Monnier L, Colette C: Glycemic variability: should we and can we prevent it? Diabetes Care 2008;31(Suppl 2):S150 S154.

29. Mohanty P, Hamouda W, Garg R, et al.: Glucose challenge stimulates reactive oxygen species (ROS) generation by leucocytes. J Clin Endocrinol Metab 2000;85:2970-2973.

30. Mohanty P, Ghanim H, Hamouda W, et al.: Both lipid and protein intakes stimulate increased generation of reactive oxygen species by polymorphonuclear leukocytes and mononuclear cells. Am J Clin Nutr 2002;75:767-772.

31. Aljada A, Mohanty P, Ghanim H, et al.: Increase in intranuclear nuclear factor kappaB and decrease in inhibitor kappaB in mononuclear cells after a mixed meal: evidence for a proinflammatory effect. Am J Clin Nutr 2004;79:682690.

32. Ghanim H, Abuaysheh S, Sia CL, et al.: Increase in plasma endotoxin concentrations and the expression of toll-like receptors and suppressor of cytokine signaling-3 in mononuclear cells after a high-fat, high-carbohydrate meal: implications for insulin resistance. Diabetes Care 2009;32:2281-2287.

33. Giacco F, Brownlee M: Oxidative stress and diabetic complications. Circ Res 2010;107:1058-1070.

34. Monnier L, Mas E, Ginet C, et al.: Activation of oxidative stress by acute glucose fluctuations compared with sustained chronic hyperglycemia in patients with type 2 diabetes. JAMA 2006;295:1681-1687.

35. Ghanim H, Sia CL, Upadhyay $M$, et al.: Orange juice neutralizes the proinflammatory effect of a high-fat, high- carbohydrate meal and prevents endotoxin increase and Tolllike receptor expression. Am J Clin Nutr 2010;91:940-949.

36. Nishikawa T, Edelstein D, Du XL, et al.: Normalizing mitochondrial superoxide production blocks three pathways of hyperglycaemic damage. Nature 2000;404:787-790.

37. Huang X, Sun M, Li D, et al.: Augmented NADPH oxidase activity and p22phox expression in monocytes underlie oxidative stress of patients with type 2 diabetes mellitus. Diabetes Res Clin Pract 2011;91:371-380.

38. Murata GH, Shah JH, Hoffman RM, et al.: Intensified blood glucose monitoring improves glycemic control in stable, insulin-treated veterans with type 2 diabetes: the Diabetes Outcomes in Veterans Study (DOVES). Diabetes Care 2003;26:1759-1763.

39. Garg S, Zisser H, Schwartz S, et al.: Improvement in glycemic excursions with a transcutaneous, real-time continuous glucose sensor: a randomized controlled trial. Diabetes Care 2006;29:44-50.

40. Kim HS, Shin JA, Lee SH, et al.: A comparative study of the effects of a dipeptidyl peptidase-IV inhibitor and sulfonylurea on glucose variability in patients with type 2 diabetes with inadequate glycemic control on metformin. Diabetes Technol Ther 2013;15:810-816.

41. Guerci B, Monnier L, Serusclat P, et al.: Continuous glucose profiles with vildagliptin versus sitagliptin in add-on to metformin: results from the randomized Optima study. Diabetes Metab 2012;38:359-366.

42. Tahrani AA, Barnett AH, Bailey CJ: Pharmacology and therapeutic implications of current drugs for type 2 diabetes mellitus. Nat Rev Endocrinol 2016;12:566-592.

43. Wang JS, Lin SD, Lee WJ, et al.: Effects of acarbose versus glibenclamide on glycemic excursion and oxidative stress in type 2 diabetic patients inadequately controlled by metformin: a 24-week, randomized, open-label, parallelgroup comparison. Clin Ther 2011;33:1932-1942.

44. Su JB, Wang XQ, Chen JF, et al.: Glycemic variability in insulin treated type 2 diabetes with well-controlled hemoglobin A1c and its response to further treatment with acarbose. Chin Med J (Engl) 2011;124:144-147.

45. Henry RR, Strange P, Zhou R, et al.: Effects of dapagliflozin (DAPA), a sodium glucose cotransporter 2 inhibitor, on 24hour glycemic control in patients with type 2 diabetes (T2D). Presented at: 76th Scientific Sessions of the American Diabetes Association; June 10-14, 2016; New Orleans, LA.

46. Simonyi G, Csitári G, Gasparics R, et al.: Silent hypoglycaemic episodes among well controlled type 2 diabetic patients treated with sulfonylureas [abstract]. Diabetologia 2015;58(Suppl 1):S465.

47. Irace C, Fiorentino R, Carallo C, et al.: Exenatide improves glycemic variability assessed by continuous glucose monitoring in subjects with type 2 diabetes. Diabetes Technol Ther 2011;13:1261-1263.

48. Mazze R, Strock E, Morgan B, et al.: Diurnal glucose patterns of exenatide once weekly: a 1-year study using continuous glucose monitoring with ambulatory glucose profile analysis. Endocr Pract 2009;15:326-334.

49. FLAT-SUGAR Trial Investigators: Glucose variability in a 26-week randomized comparison of mealtime treatment with rapid-acting insulin versus GLP-1 agonist in participants with type 2 diabetes at high cardiovascular risk. Diabetes Care 2016;39:973-981.

50. Frías JP, Nakhle S, Ruggles JA, et al.: Exenatide once weekly improved 24-hour glucose control and reduced glycemic variability in metformin-treated patients with type 
2 diabetes: a randomized, placebo-controlled trial. Diabetes Obes Metab 2016;19:40-48.

51. Vora JP, Malloy J, Zhou M, et al.: 997-P Daily blood glucose variability with exenatide once weekly vs. basal insulin in 3 RCTs [abstract]. Diabetes 2014;63:A256.

52. Holst JJ, Buse JB, Rodbard HW, et al.: IDegLira improves both fasting and postprandial glucose control as demonstrated using continuous glucose monitoring and a standardized meal test. J Diabetes Sci Technol 2016;10:389-397.

53. Ma Z, Chen R, Liu Y, et al.: Effect of liraglutide vs. NPH in combination with metformin on blood glucose fluctuations assessed using continuous glucose monitoring in patients with newly diagnosed type 2 diabetes. Int J Clin Pharmacol Ther 2015;53:933-939.

54. Lane W, Weinrib S, Rappaport J, et al.: The effect of addition of liraglutide to high-dose intensive insulin therapy: a randomized prospective trial. Diabetes Obes Metab 2014; 16:827-832.

55. Mori Y, Taniguchi Y, Sezaki K, et al.: Liraglutide narrows the range of circadian glycemic variations in Japanese type 2 diabetes patients and nearly flattens these variations in drug-naive type 2 diabetes patients: a continuous glucose monitoring-based study. Diabetes Technol Ther 2011;13: 1139-1144.

56. Nagakura J, Yamakawa T, Taguri M, et al.: Effects of exenatide and liraglutide on 24-hour glucose fluctuations in type 2 diabetes. Endocr J 2016;63:239-247.
57. Jendle J, Testa MA, Martin S, et al.: Continuous glucose monitoring in patients with type 2 diabetes treated with glucagon-like peptide-1 receptor agonist dulaglutide in combination with prandial insulin lispro: an AWARD-4 substudy. Diabetes Obes Metab 2016;18:999-1005.

58. Makdissi A, Ghanim H, Vora M, et al.: Sitagliptin exerts an antinflammatory action. J Clin Endocrinol Metab 2012; 97:3333-3341.

59. Chaudhuri A, Ghanim H, Vora M, et al.: Exenatide exerts a potent antiinflammatory effect. J Clin Endocrinol Metab 2012;97:198-207.

60. Godinho R, Mega C, Teixeira-de-Lemos E, et al.: The place of dipeptidyl peptidase- 4 inhibitors in type 2 diabetes therapeutics: a "me too" or "the special one" antidiabetic class? J Diabetes Res 2015;2015:806979.

61. Minambres I, Perez A: Is there a justification for classifying GLP-1 receptor agonists as basal and prandial? Diabetol Metab Syndr 2017;9:6.

Address correspondence to:

Paresh Dandona, MD, PhD

$M O B$, Erie County Medical Center

462 Grider Street

Buffalo, NY 14215

E-mail: pdandona@kaleidahealth.org 